\title{
104. Specific Changes in the Distribution Pattern of Silver-stained Nucleolar Organizer Regions in Chemically Transformed Rat Glial Cells
}

\author{
By Kyoko Kano-Tanaka,*),**) Hiroko Fukami,*) Taiji Kato,***) \\ Jin-ichi ITo,***) and Tatsuya TANAKA*),**)
}

(Communicated by Sajiro Makino, M. J. A., Dec. 12, 1986)

Introduction. The previous study in which were investigated the cytogenetics of rat glial cells in seven MSV-transformed cell lines, showed that not only specific chromosome changes but also consistent changes in the distribution pattern of silver-stained nucleolar organizer regions (Ag-NORs) were associated with malignant transformation in vitro.2) Several workers have reported the alteration in rRNA gene activity and/or the amount of rDNA of the NORs of malignant rat chromosomes and suggested the importance of rRNA gene amplification in the origin or progression of tumors.5),6),8) What the relation between karyotypic changes and changes of NOR-activity existed in the association with malignancy, however, has remained unclear. Two new GA cell lines derived from chemically transformed rat glioblasts ${ }^{3)}$ appeared to be favorite materials to provide an additional evidence for a solution of this matter. We described G-banded karyotypes of these cells in a separated paper. ${ }^{+)}$The present one deals with their Ag-NOR patterns and discusses the results by combining with the previous data.

Materials and methods. Cells, rat strains and methods for G-banding chromosome analysis were described.4) For the NOR examination, the slides prepared with the same cell samples for G-banding were given Ag-As staining. ${ }^{1)}$

Results. The Ag-NOR chromosomes in the normal rat cells have been known to be three chromosome pairs, nos. 3, 11 and 12 (Fig. 1). The distribution pattern of Ag-NOR sites in glioblasts, however, varied between two given strains, WKA and BDIX. The former rat had Ag-NORs on three pairs (Fig. 1a) whereas the latter one did not show any detectable grains on chromosome no. 12 (Fig. 1c). The Ag-NOR frequency of each chromosome pair also varied strain to strain, being chromosomes nos. 3, 11 and 12 in WKA whereas chromosomes nos. 11 and 3 in BDIX, in order of Ag-positive value (Table I). The size or staining intensity of Ag-NORs correlated well with their frequency.

The distribution patterns of Ag-NORs in two malignant glial cell lines, GA1 and GA2, on the other hand, were characterized by showing a difference from those of the respective parental strain (WKA for GA1 and BDIX for GA2) but resulting in the similar pattern each other (Table I). Changes in the frequency were particularly remarkable for the chromosome no. 12 dominance and either the 464.

*) Laboratory of Cell Biology, Aichi Cancer Center Research Institute, Nagoya

**) Present address: Division of Neurobiology, Awazu Neuro-Sanatorium, 54-3 Kitashinden, Kataharacho, Gamagori 443-01.

***) Department of Biochemistry, Nagoya City University Medical School, Nagoya 467. 


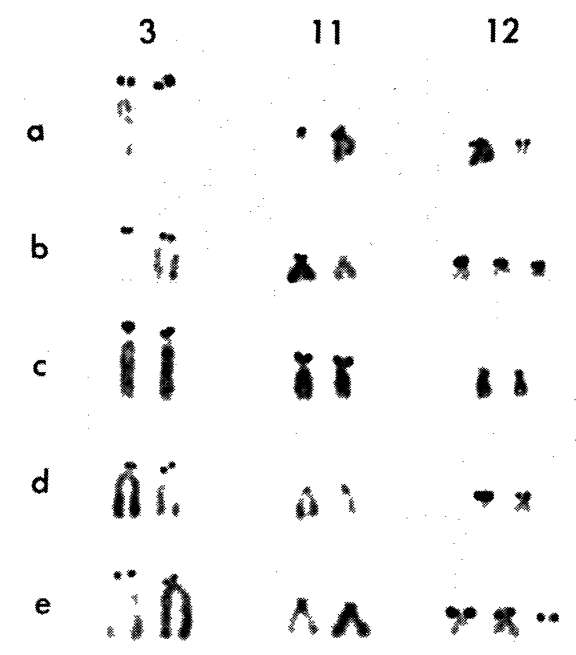

Fig. 1. Ag-NOR patterns in chromosomes nos. 3, 11 and 12 of WKA glioblasts (a), GA1 (b), BDIX glioblast (c) and GA2 (d, e).

Table I. Frequency of Ag-NORs in normal and malignant glial cells of WKA and BDIX rats

\begin{tabular}{|c|c|c|c|c|c|c|c|}
\hline \multirow[t]{2}{*}{ Cell } & \multirow{2}{*}{$\begin{array}{c}\text { Rat } \\
\text { strain }\end{array}$} & \multirow{2}{*}{$\begin{array}{l}\text { Chromosome } \\
\text { number }\end{array}$} & \multicolumn{3}{|c|}{$\begin{array}{c}\text { Parcentage of } \\
\text { Ag-positive chromosomes }\end{array}$} & \multirow{2}{*}{$\begin{array}{c}\text { Total numbers } \\
\text { of Ag-NORs/ } \\
\text { metaphase } \\
\text { (mode) }\end{array}$} & \multirow{2}{*}{$\begin{array}{l}\text { No. of } \\
\text { cells } \\
\text { scored }\end{array}$} \\
\hline & & & 3 & 11 & 12 & & \\
\hline glioblast & WKA & 42 & 100 & 70 & 43 & $3-6(5)$ & 15 \\
\hline glioblast & BDIX & 42 & $92 *$ & 97 & 0 & $2-4(4)$ & 30 \\
\hline GA1 & WKA & $43 * *$ & $58^{*}$ & 5 & $141^{*}, * * *$ & $3-6(5)$ & 64 \\
\hline \multirow[t]{2}{*}{ GA2 } & BDIX & 42 & 70 & 4 & 95 & $2-5(4)$ & 80 \\
\hline & & $43^{* * *}$ & 70 & 0 & $130 *$, **** & $3-5(4)$ & 10 \\
\hline
\end{tabular}

* High incidence of heteromorphic Ag-NORs among homologs. ** Karyotype with trisomy 12 . $* * *$ Value is attributed to trisomy.

reduction or missing of chromosome no. 11 as contrasted with the normal value. Even more, extraordinarily high values of chromosome no. 12 in cells with 43 chromosomes were attributable to trisomy 12 bearing Ag-NORs. In many cases, one of trisomic NORs was stained most intensely, as seen in Figs. $1 \mathrm{~b}$ and 1c-e. Thus the heteromorphic sizes and/or the stainability of chromosome no. 12 were also evident. There was no significant increase in the total number of Ag-NORs per metaphase.

Discussions. It is striking that Ag-staining karyotypes of GA cells demonstrated malignancy-associated, specific changes in the distribution pattern of Ag-NORs. The similar results have been obtained from all of seven MSVtransformed cell lines (2GT, 3GT, 5GT and C6V1-4) of WKA and Wistar origin.2) Moreover, even GA2 cells with the normal karyotype exhibited wholly the malignant pattern and that was in a good agreement with the phenomenon found in C6 cells.2) Sugiyama et al. ${ }^{8}$ ) have reported a significant increase in the gene dosage of nucleolar cistrons (rDNA) in rat leukemic cells with the normal 
karyotype. This is suggestive of a possible increase in the NOR function for the present case.

Another interesting finding in GA cells was that such an abnormal pattern common to two lines was resulted from cells originally showing the distinct strain-specific difference of Ag-NORs. The strain-specific patterns of Ag-NORs characterizing 16 inbred strains of the rat, including WKA but not BDIX, have been recently reported. ${ }^{7}$ ) Thus, our combined data on Ag-NORs from the prior and present studies clearly indicated that the NOR-pattern in malignant glial cells transformed in vitro was found to be tumor-specific regardless of the type of oncogenic agents, host strains and even the karyotype. In addition, the evidence that subtle chromosome changes (functional or genic) without karyotypic changes in the malignant transformation are consistently recognizable at the level of rRNA gene activity on the NORs strongly suggested the particular importance of the aberrant NOR function for an acquisition of the malignancy at the initial step of oncogenesis. The mechanism of these changes therefore has to be elucidated.

Summary. Ag-NORs in two GA cell lines, derived from chemically transformed glioblasts of WKA and BDIX rats were investigated. The distribution patterns of Ag-NORs among chromosomes nos. 3, 11 and 12 in normal glioblasts varied between two rat strains. The Ag-NOR pattern in GA cell lines, on the other hand, was different from the respective normal one but it was similar between two cells. The identical NOR abnormalities have also been observed in the virustransformed glial cells. The results combined from both studies showed that specific changes in the Ag-NOR pattern are associated with in vitro transformation of rat glioblasts regardless of the type of the carcinogenic agent, rat strain as well as the karyotype.

Acknowledgment. We are grateful to Emeritus Professor Dr. Sajiro Makino, M. J. A., the Hokkaido University, for his critical reading of this manuscript.

\section{References}

1) Goodpasture, C., and Bloom, S. E. (1975): Chromosoma, 53, 37-50.

2) Kano-Tanaka, K., and Tanaka, T. (1982) : Int. J. Cancer, 30, 495-501.

3) Kano-Tanaka, K. et al. (1986): Proc. Japan Acad., 62B, 109-112.

4) Kano-Tanaka, K. et al. (1986): ibid., 62B, 395-397.

5) Miller, D. J. et al. (1979): Chromosoma, 71, 183-195.

6) Murano, S. et al. (1982) : Cancer Genet. Cytogenet., 6, 303-312.

7) Sasaki, M., Nishida, C.,, and Kodama, Y. (1986): Cytogenet. Cell Genet., 41, 83-88.

8) Sugiyama, T. et al. (1980): Gann, 71, 372-380. 